\section{ASNR 2020}

Purpose

- To familiarize the audience with the concepts of deep learning for image classification.

- To show the audience how to make an actual model to classify CT images for the prediction of intracranial hemorrhage.
Achint Singh MD, Kal Clark, MD

UT Health San Antonio

\section{Introduction}

\section{What is Deep Learning?}

Subset of machine learning based on convolutional neural networks where the model learn the relevant features of the data by themselves

What is a neural network?

Neural network has multiple layers and each layer passes the processed information to the next layer with increasing level of complexity
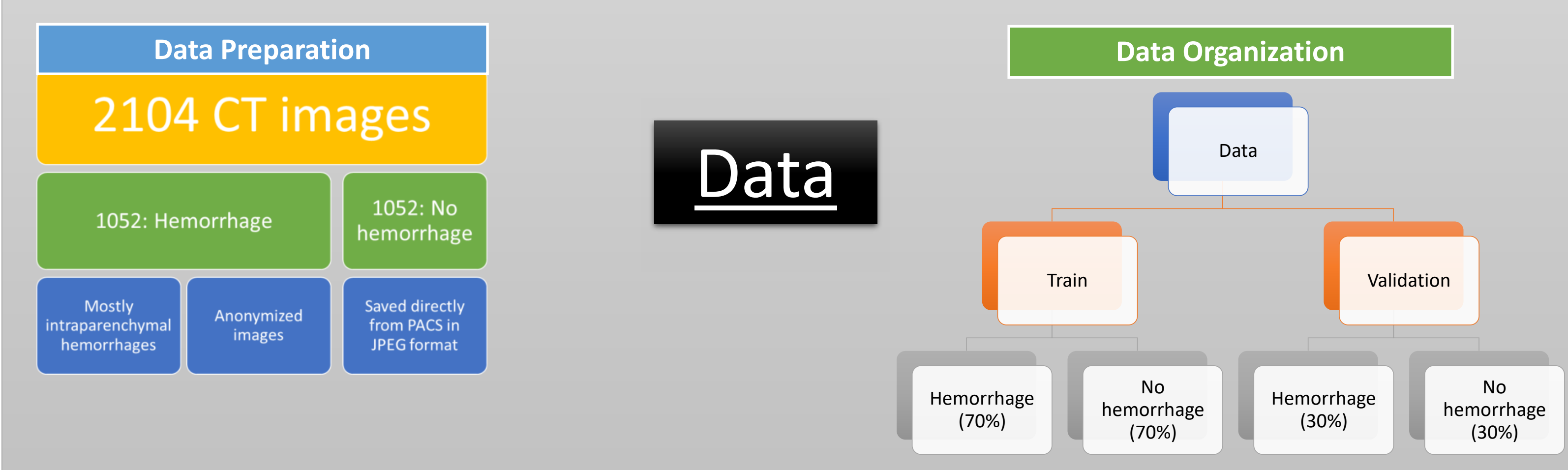

\title{
Model Results
}

\section{How to do it?}

\section{Hardware}

- PC with GPU card

\section{Software}

- Fastai version 1 (https://docs.fast.ai/): Freely available - Based on PyTorch

\section{Approach}

- Transfer Learning: Use of model (Resnet50) already heavily tested on other images

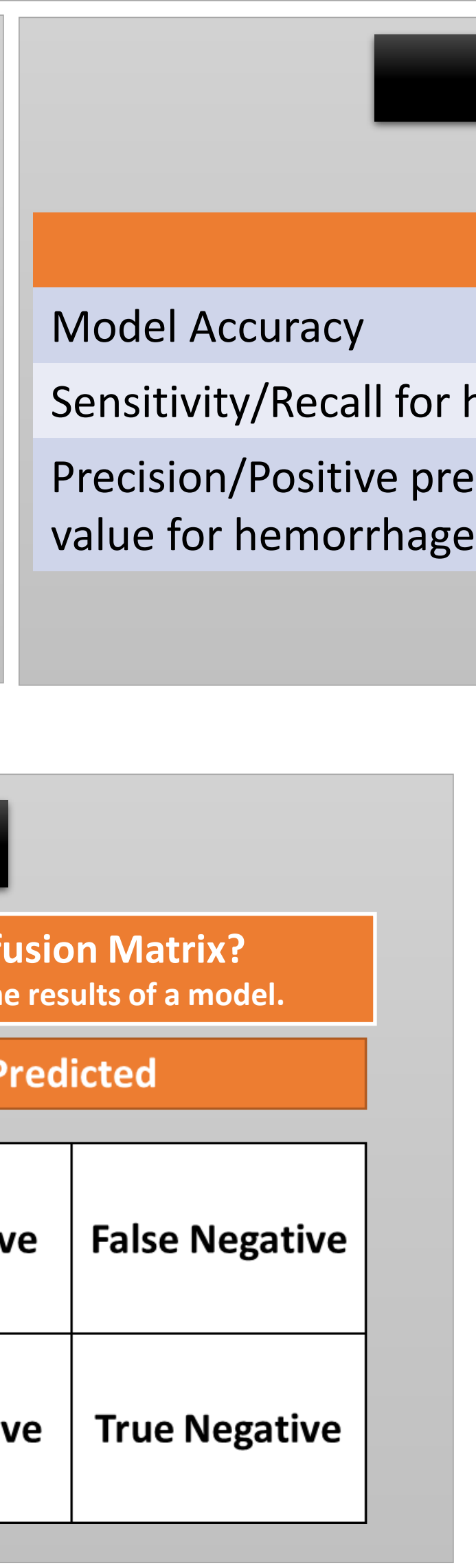

What is Confusion Matrix? A way to analyze the results of a model.

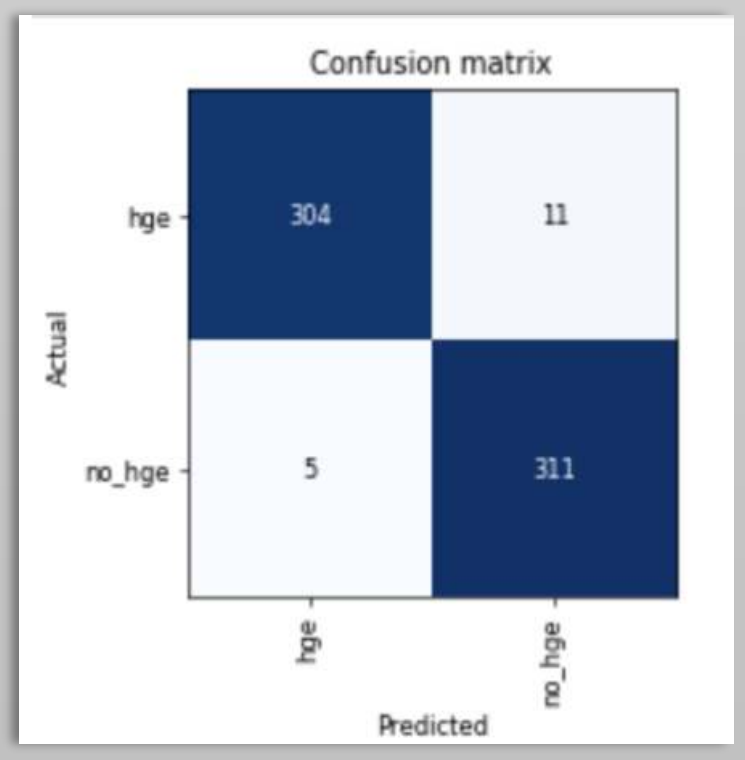

\begin{tabular}{|c|c|c|}
\hline & \multicolumn{2}{|c|}{$\begin{array}{l}\text { What is Confusion Matrix? } \\
\text { A way to analyze the results of a model. }\end{array}$} \\
\hline & \multicolumn{2}{|c|}{ Predicted } \\
\hline & True Positive & False Negative \\
\hline & False Positive & True Negative \\
\hline
\end{tabular}

\section{References}

https://docs.fast.ai/index.html

Saba L, Biswas M, Kuppili V, et al. The present and future of deep learning in radiology. Eur J Radiol 2019;114:14-24.

\section{Model Evaluation}

Copyright $\odot 2020$ Achint Singh, Kal Clark singhak@uthscsa.edu

\section{Result}

\section{$97.4 \%$}

$96.5 \%$

$98.4 \%$

\section{Deployment}

\begin{tabular}{|l|}
\hline \multicolumn{1}{|c|}{ Model Deployment } \\
\hline - Post training model was saved \\
in a GitHub repository \\
- Online deployment was done \\
using Render.com platform \\
- Link for final model: \\
https://www.neuroradiologye \\
ducation.com/ct-hemorrhage \\
\hline
\end{tabular}

\section{Acknowledgment}

Fast.ai team (Jeremy Howard, Rachel Thomas and Sylvain Gugger) for providing excellent software and online videos for everyone for free. 